\title{
AN APPRECIATION OF JEAN BOURGAIN'S WORK
}

\author{
PETER SARNAK
}

Jean Bourgain viewed himself as an "analyst", and as the record shows he was uniquely gifted as such, and much more. Analytic, combinatorial, and probabilistic reasoning is at the heart of many central problems of modern mathematics and its applications, and these naturally attracted Jean's attention. The combination of his brilliance, his thirst to solve long-standing problems, and his many fruitful collaborations led him to transformative contributions in a striking number of areas. Jean's research and its impact remind one of the great Russian analyst Kolmogorov. It is said that Kolmogorov made major contributions to all fields except number theory. A list of areas to which Jean made decisive contributions include functional analysis, harmonic analysis, probability theory, ergodic theory, partial differential equations, mathematical physics, number theory, group theory, and theoretical computer science.

It is impossible in a single volume, let alone an issue of the Bulletin of the American Mathematical Society, to give anything like a comprehensive account of Jean's mathematical achievements. Gathering his over 500 (and counting) publications in a collected works would be physically impossible. Fortunately, Jean was very purposeful and proactive in preparing his papers for publication, and almost all of these are in print and are accessible. He made sure that anyone committed to understanding and using his work would have it available.

The four articles in this issue give very clear and insightful accounts of some highlights of Jean's work in functional analysis (Ball), harmonic analysis (Demeter), dispersive partial differential equations (Kenig), and a deconstruction of some of the techniques that Jean invented (Tao). The reports on some of Jean's earlier works highlight how his breakthroughs impacted and reshaped each of these fields. In many cases his resolution of a long-standing problem introduced fundamental new tools, insights, and viewpoints allowing others to achieve further lofty goals. Many view Jean primarily as a problem solver, perhaps because that is the impression he liked to give. The first solution of a fundamental problem almost always comes with new tools, insights, and theory, so naturally Jean was both a problem solver and a theory builder of the highest calibre. The report on Jean's more recent work on $l^{2}$ decoupling (Demeter) explains a striking application resolving a long-standing problem in analytic number theory-the Vinogradov mean value conjecture. Jean's expectation that the decoupling theory will yield much more has certainly materialized recently, and it will no doubt continue to do so.

I was fortunate enough to be Jean's colleague and collaborator and to witness a number of his breakthroughs first hand. I mention a few of these that I am particularly fond of and which are not discussed in the four reports. The first is around the sum-product phenomenon, as Jean liked to call it, and its applications. In Bou03. Jean gave a proof of a local version of the Erdös-Volkmann conjecture. 
The conjecture itself asserts that the Hausdorff dimension of a Borel measurable subring of the reals must be either 0 or 1, and it was proven by Edgar and Miller, shortly before Jean, and by different methods. Together with Katz and Tao, Jean followed this up by proving a finite field analogue [BKT04]. This fundamental result measures the disjointness of the multiplication and addition operations in a finite field, and is known as the sum product theorem. Anticipating its potential, Jean proceeded to develop an elaborate theory which, when combined with other tools, led to many applications. These include his bounds for character sums over finite fields Bou05] in cases where the usual tools from algebraic geometry, namely the Weil conjectures, have little to offer. The sum product theorem as well as a number of other very novel ideas (for example, the flattening lemma) were used in his breakthrough with Gamburd establishing the critical expansion property for the finite simple groups $\operatorname{PSL}\left(2, \mathbb{F}_{p}\right)$ BG08a, while the full force of the local ring conjecture mentioned above was a key input in their proof of the expansion property in the much more difficult case of the classical groups $\mathrm{SU}(n)$ [BG08b]. These expansion properties have been applied decisively in problems in group theory, number theory, as well as in theoretical computer science. The story is similar to the other ones told about Jean - after working intensively for a number of years on a topic, he would transform its basic theory, tool kit, and dramatically extend its boundaries.

The final work that I mention is Jean's recent paper with Semyon Dyatlov BD18. Semyon works in microlocal analysis, and he and his collaborators had reduced some very interesting and difficult problems about localizations of eigenfunctions on hyperbolic surfaces to a harmonic analysis question. I remember Semyon explaining the problem to me in my office and providing ample numerical evidence for what he believed to be true. It was quickly apparent that I had no ideas to offer, and it was equally clear to both of us as to whose office he should go to next. I didn't hear from either of them for some time, but then as often was the case with Jean when he had progress to report, I got a short and animated email from him saying that they had solved the problem. The fractal uncertainty principle that they proved was an instant classic, both as a powerful new tool in microlocal analysis and as an independently interesting result in harmonic analysis.

In conclusion let me add that Jean understood that the broad impact of his work was only possible through his many collaborations. His was very appreciative of his coauthors and correspondingly generous with his ideas. As far as I can tell, all of his collaborators are equally appreciative of their opportunity to partner with such a singularly brilliant mathematician.

\section{REFERENCES}

[Bou03] J. Bourgain, On the Erdös-Volkmann and Katz-Tao ring conjectures, Geom. Funct. Anal. 13 (2003), no. 2, 334-365, DOI 10.1007/s000390300008. MR1982147

[Bou05] J. Bourgain, Mordell's exponential sum estimate revisited, J. Amer. Math. Soc. 18 (2005), no. 2, 477-499, DOI 10.1090/S0894-0347-05-00476-5. MR2137982

[BD18] J. Bourgain and S. Dyatlov, Spectral gaps without the pressure condition, Ann. of Math. (2) 187 (2018), no. 3, 825-867, DOI 10.4007/annals.2018.187.3.5. MR3779959

[BG08a] J. Bourgain and A. Gamburd, Uniform expansion bounds for Cayley graphs of $\mathrm{SL}_{2}\left(\mathbb{F}_{p}\right)$, Ann. of Math. (2) 167 (2008), no. 2, 625-642, DOI 10.4007/annals.2008.167.625. $\operatorname{MR} 2415383$ 
[BG08b] J. Bourgain and A. Gamburd, On the spectral gap for finitely-generated subgroups of SU(2), Invent. Math. 171 (2008), no. 1, 83-121, DOI 10.1007/s00222-007-0072-z. MR2358056

[BKT04] J. Bourgain, N. Katz, and T. Tao, A sum-product estimate in finite fields, and applications, Geom. Funct. Anal. 14 (2004), no. 1, 27-57, DOI 10.1007/s00039-004-0451-1. MR2053599

School of Mathematics, Institute for Advanced Study, Princeton, New Jersey 